\title{
Fractal Dimension Calculation for the General Shapes of Geographical Maps and Closed Curves
}

\author{
Rayan Yousif Alkhayat \\ Basil Younis Thanoon \\ rayan@uomosul.edu.iq \\ basilyt@uomosul.edu.iq \\ College of computer sciences and mathematics \\ University of Mosul, Iraq
}

Received on: 10/03/2004

Accepted on: 05/04/2005

\begin{abstract}
This research includes building a software to calculate the Fractal Dimension of the general shapes of the borders of geographical maps as well as closed curves. Two methods are adopted for calculating fractal dimension: the first is the well-known Box Counting method and the second is Two-dimension Variation method, some treatments of the two methods are performed in order to qualify them for practical application and fitness for the use in digital images which are used for the representation of borders of maps and closed curves. Matlab 6.5 programming language was used for the implementation of this work, the map of the republic of Iraq was used as a case study for the given work.
\end{abstract}

Keywords: Fractal Geometry, Fractal Dimension, Geographical Maps.

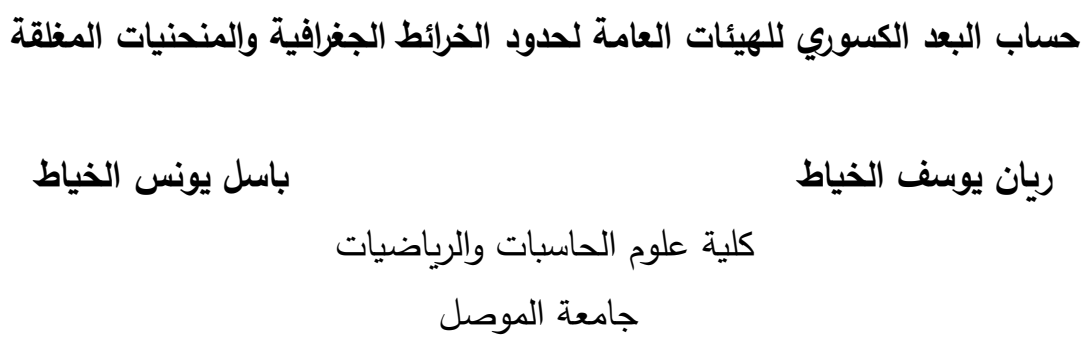

تاريخ قبول البحث: 2005/04/05

تاريخ استلام البحث: 2004/03/10

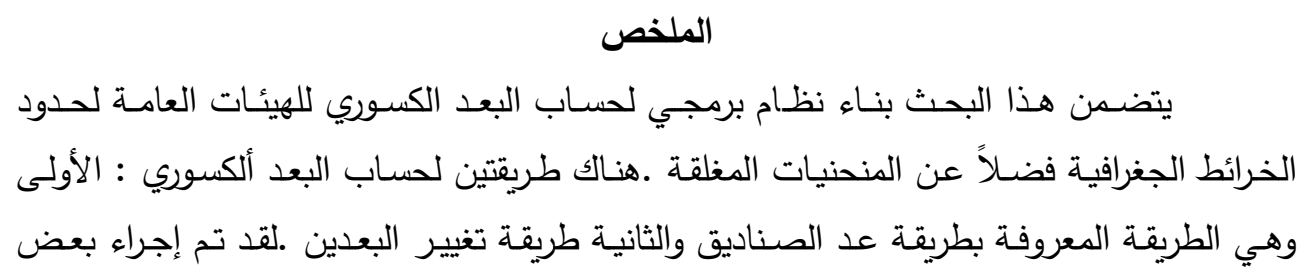


المعالجات للطريقتين لغرض تأهيلهما للتطبيق العملي وملاءمتهما للاستخدام على الصور الرقمية

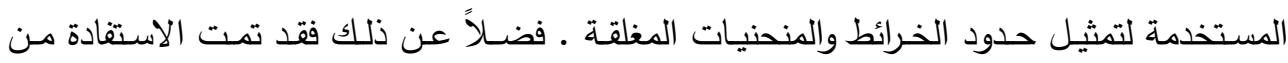
التقطيع ألكسوري لغرض حساب طول الحدود للخرائط مقاسا بالكيلومتر وكذلك للمنحنيات المغلقة مقاسا بالسنتيمتر .لقد استخدمت لغة البرمجة الخاصة بتطبيق 6.5 Matlab لبرمجة النظام تحت

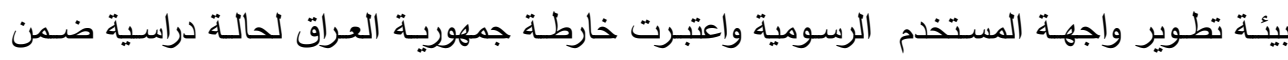
الحالات التي أخذها النظام المعد. الكلمات المفتاحية: الهندسة الكسورية, البعد الكسوري, الخرائط الجغرافية.

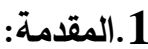

أن الخريطة الجغرافية هي لغة عالمية وظيفتها حفظ التراث الجغرافي ونقله وتعد وسيلة إيضاح ضرورية ـ و من المعلوم بان حدود الخرائط الجغرافية هي الأساس المعتمد لأجل تحديد

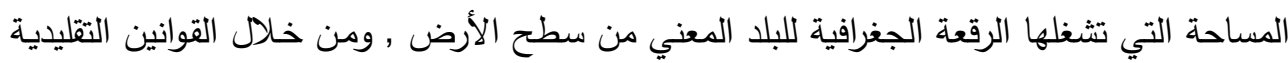

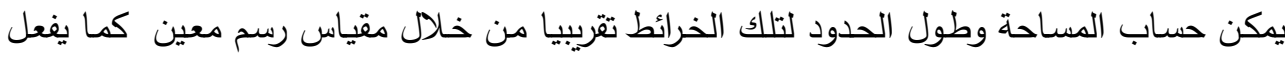
ذلك علماء المساحة و المختصون بالجغرافية . لقد كان ,حتى وقت قريب تعتمد التخمينات والحسابات المقترنة بالحدس في إيجاد الثكل التقريبي للهيئة التي يعتمد عليها في رسم الخارطة الجغرافية لرقعة ما على سطح الكرة الأرضية .

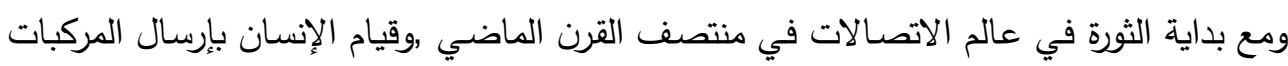

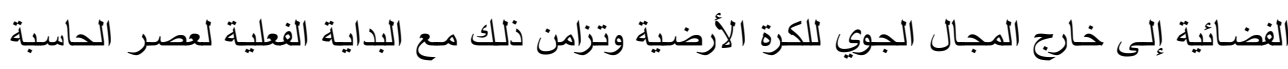

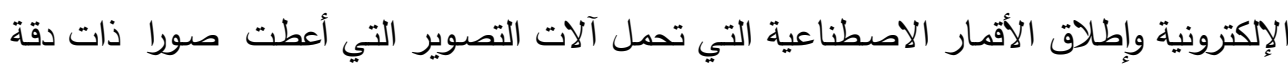

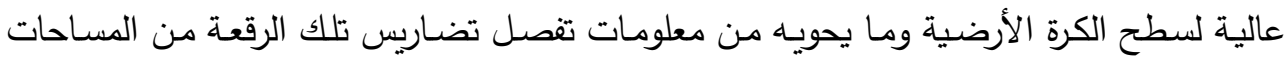

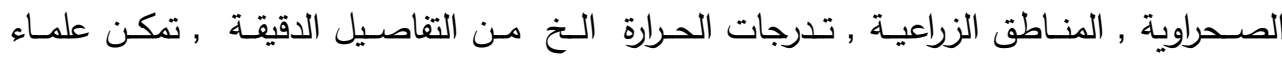
الجغرافية ضمن مجال التحسس النائي من الحصول على بلى صور دورات دقيقة مدعومة بالحاسب الآلي يشار

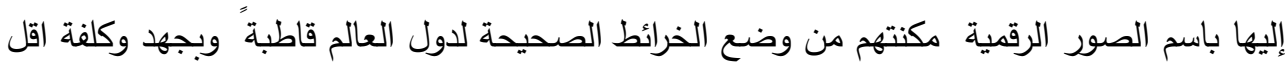
مما في الطرائق التقليدية.

يتضمن موضوع البحث استخدام بعض أساليب الهندسة الكسورية لغرض حساب البعد

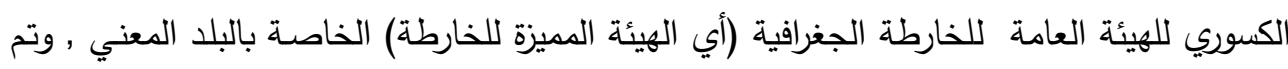
إجراء العمليات لأجل حساب البعد الكسوري للخرائط على صور رقمية لحدود خرائط معينة لمجموعة لهئة من الدول العربية , ضمن النوع الثالث من الصور الرقمية , وبتدرجات لونية محدودة ب256 لوناً 
فقط , ضمن صيغة الخزن لملفات (BMP), وذلك لكي تكون الحسابات دقيقة للبيانات المأخوذة

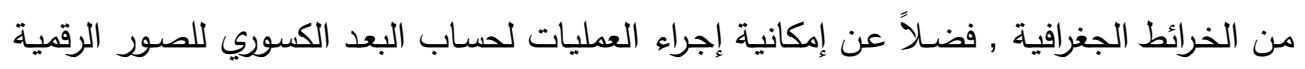

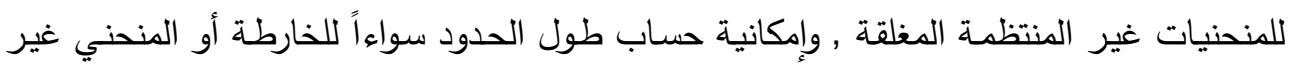

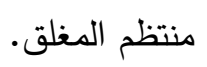

\section{Fractal Geometry الهندسة الكسورية}

ان الهندسة الكسورية او (هندسة الكسوريات) هي منطلق جديد في إطار الهندسة العامة

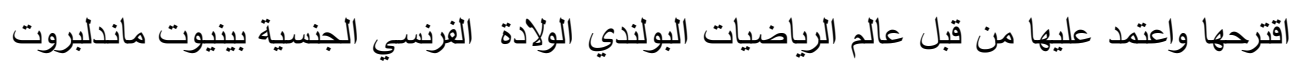

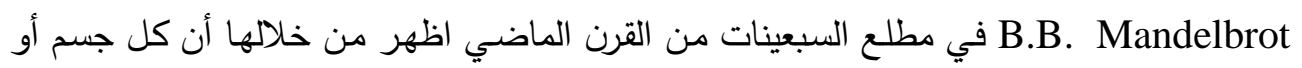
شكل في الطبيعة, الذي يعد غير منتظم من وجهة نظر الهندسة التقليدية, هو جسم أو شكل منتظم

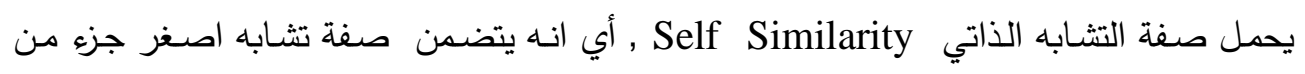

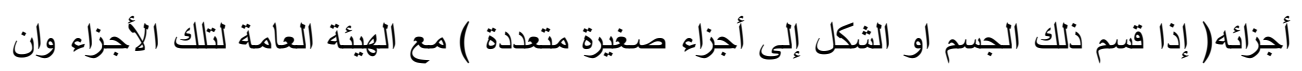

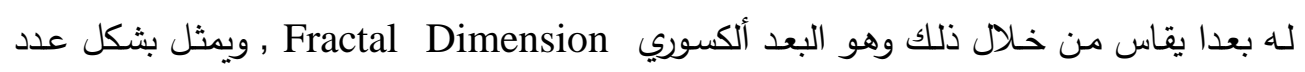

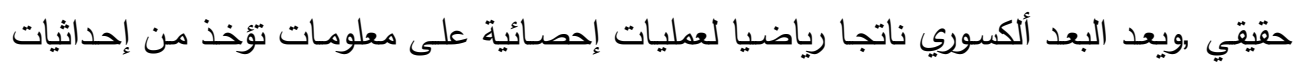

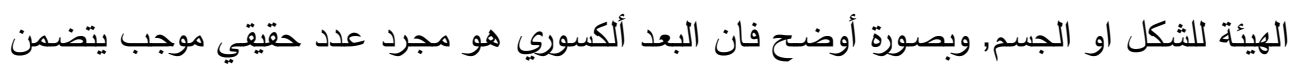

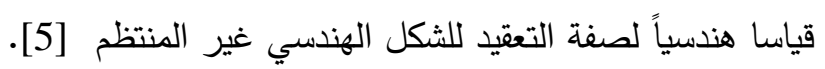
ولكون الخرائط الجغرافية هي أشكال شاذة غير منتظمة (منحني مغلق غير منتظم ) وليس

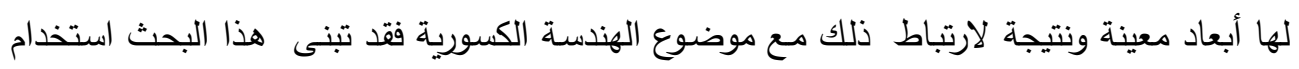

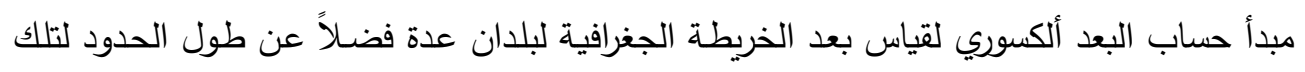
• البلدان

على مدى مائتي عام مضت كانت نظريات المنحنيات والسطوح تتطور في إطار البعدين والثثلاثة الأبعاد فأكثر , إن هكذا أثكال تمتلك في الغالب هياكل خارجية معقدة , وإذا نظرنا إليها

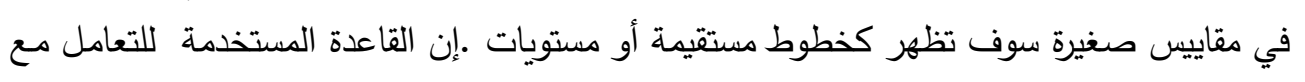

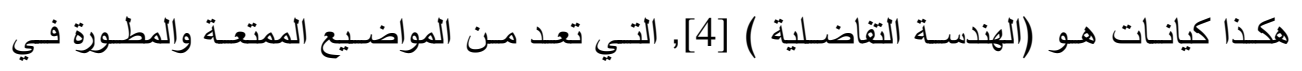
الرياضيات.

على الجانب الآخر فـان الأثكال الطبيعيـة كالسواحل , الجبـال , الأثجار , الأوراق ,

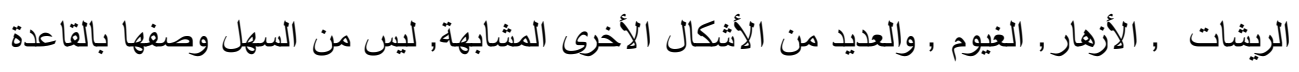

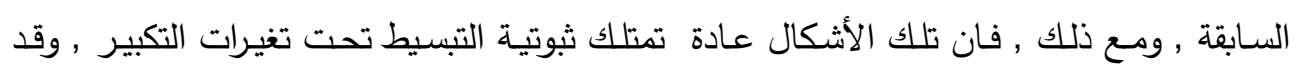


وصف هذه الأشكال العلماء حديثا لان هذا العلم هو فرع جديد من الرياضيات , لـه القدرة على

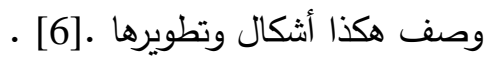

اقترح ماندلبروت وجود عناصر هندسية سماها بألكسوريات

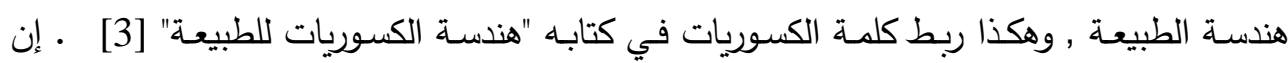
الابتكار ألكسوري الذي اكتشفه ماندلبروت هو شيء مضاد للملاسة (Smoothness) فعلى الرغم

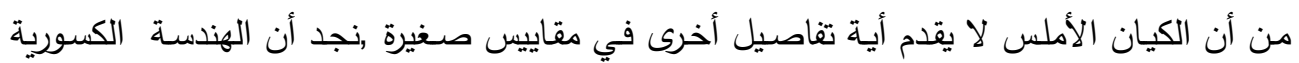
تقدم تقاصيل غير منتهية في جميع المقاييس بدون الاهتمام الى كيف يكون مقدار الصغر أو الكبر الكبر

إن الكيان يمثل دائما كسلسة او مقطع من السلسلة, من النقاط , إذا أخذنا خطا ذا طول

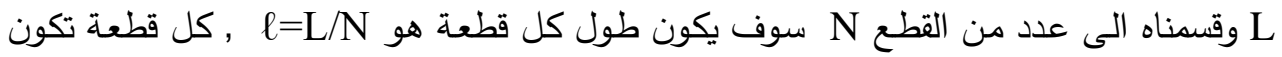

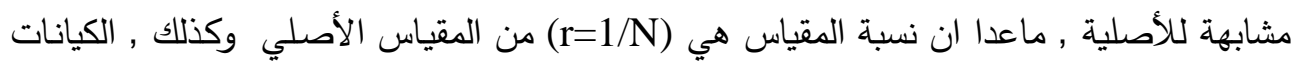
ثنائية الأبعاد , كالمساحة المربعة او السطح , ممكن ان تقسم الى N من الأجزاء المتماثلة للكيان

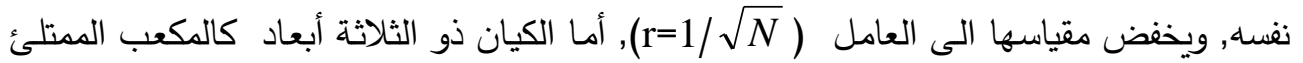
فيمكن تقسيمه الى N N N من المكبات الصغيرة التي يخفض مقياسها بالنسبة(

يوضح الثكل (1) [5].

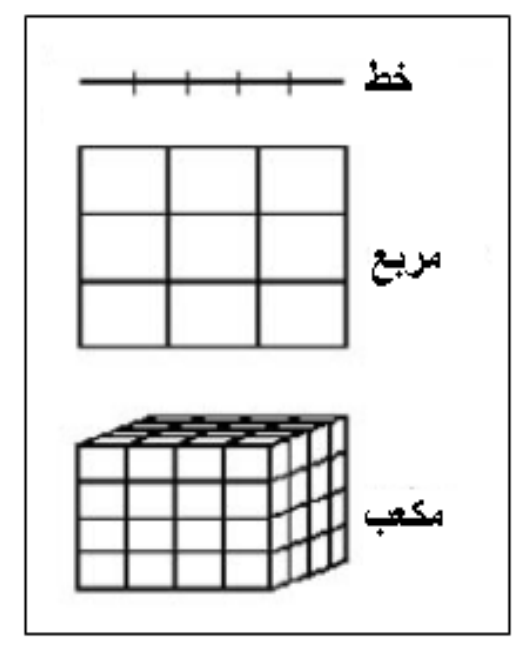

الشكل (1) صيغ التقطيع للكيانات 
من خلال التشابه الذاتي فان التعميم الى البعد الكسوري يكون قد اصبح واضحا فان

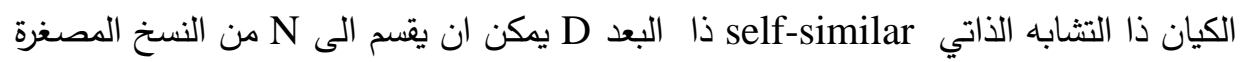

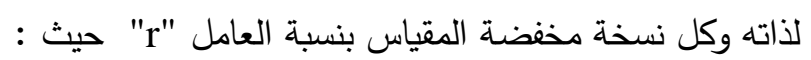

$\mathrm{r}=\frac{1}{\sqrt[D]{N}}$

$\mathrm{N}=\frac{1}{r^{D}}$

على العكس مما سبق , إذا أعطي كيان ذو صفة تثابه ذاتي مؤلف من N من الأجزاء بالنسبة r من الكل , فان بعد التثابه الذاتي له أو ألكسوري يحسب من دل خلال :

$\mathrm{D}=\frac{\log (N)}{\log \left(\frac{1}{r}\right)}$,

إن البعد الكسوري يختلف عن الأبعاد الاقليدية الظاهرية المألوفة , ذلك لكونه لا يحتاج

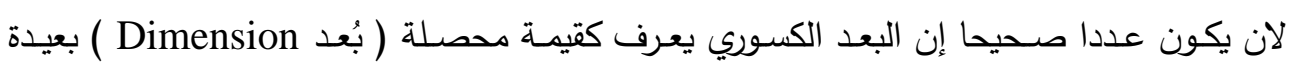
الاعتماد عن المقياس الذي يغطيها , مـع ذلك فان البعد يتأثر في التطبيق اعتمـاداً على العينـة

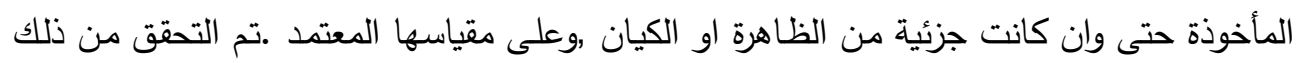

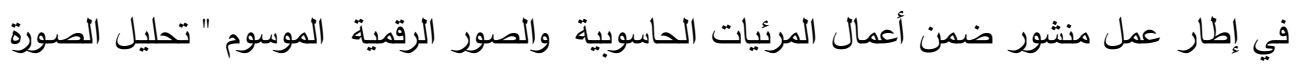
باستخدام البعد الكسوري " افترض ان البعد الكسوري محسوب ومستقل عن المقاييس , متجـاهلا

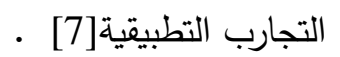

إذا كانت S تمثل مجموعة جزئية محدودة Bounded subset في وكانت N(r) تمثل عدد

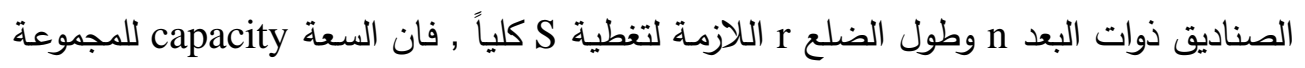
أو "بعدها الهاوسدروفي Hausedroff Dimension " يرمز لها ب(2) S

$d_{H}(S)=\lim _{r \rightarrow 0} \frac{\log [N(r)]}{\log (1 / r)}$.

ومن المعروف أيضا بان سعة المجموعة تكون مساوية لبعدها الكسوري [1] , بعبارة اخرى فان

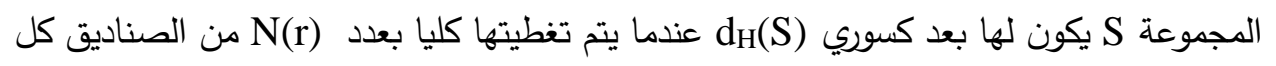
منها ذات طول ضلع r , إذ أن r هو عدد حقيقي موجب وصغير جداً وان [2] : 
$\mathrm{N}(\mathrm{r})=\mathrm{k}(1 / \mathrm{r})$

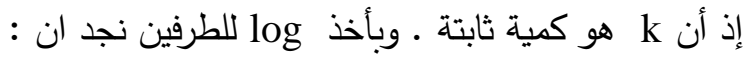

$\log [\mathrm{N}(\mathrm{r})]=\mathrm{dH}(\mathrm{S}) \log (\mathrm{k})+\mathrm{d}_{\mathrm{H}}(\mathrm{S}) \log (1 / \mathrm{r})$

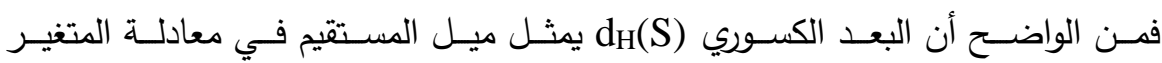

Log[N(r)]

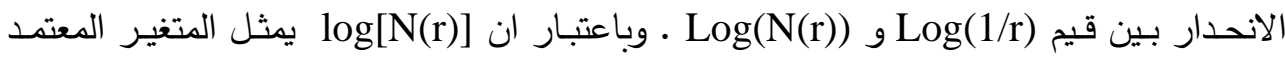

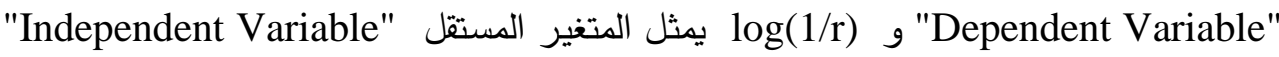
فان معادلة الانحدار الخطية يمكن ملاءمتها ولها الثكل العام الأتي عند كل تغير لقيمة (r) : $\log [\mathrm{N}(\mathrm{r})]=\mathrm{a}+\mathrm{b} \log (1 / \mathrm{r})$

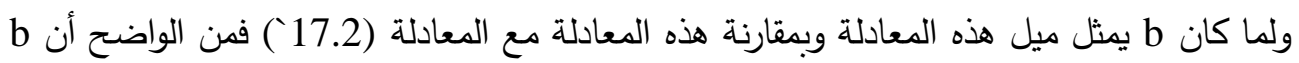
$b=\frac{\log [N(r)]-a}{\log (1 / r)}$ يمثل القيمة التقديرية للبعد ألكسوري , وحسب المعادلة التالية :

$$
\text { وان a هي كمية ثابتة . }
$$

ومن الطرائق الثنائعة لحساب البعد الكسوري هي طريقة عد الصناديق إذ تستخدم هذه

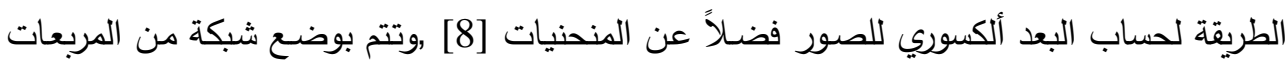

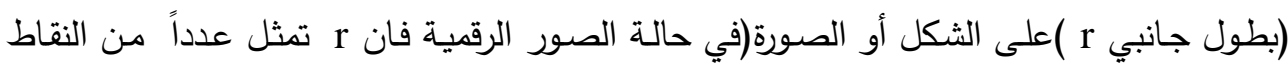
الصـورية Pixels), ثم حسـاب عدد المربعات (N) التي تضـم الثكل الذي يـراد حسـاب بعـده ألكسوري, ثم نكرر العملية بقيم مختلفة من معامل التصغير لقيمة r r r بعدها نحسب البعد

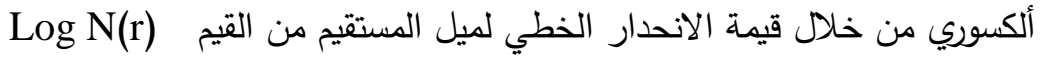
مع Log r مسب الخوارزمية آلاتية :

3. الخوارزمية (1) خوارزمية حساب البعد ألكسوري - طريقة عد الصناديق:

1 IM قراءة محتويات الصورة في مصفوفة ثنائية ولتكن

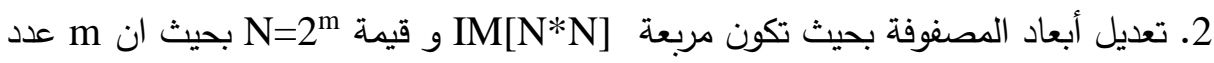

3.إنثاء التكرار في المتغير p وليكن p=0

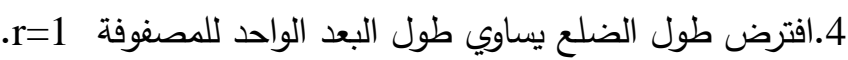

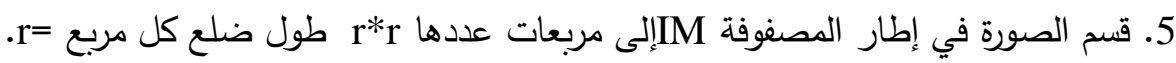




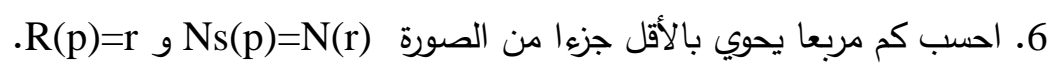

7. احسب قيمة اللوغاريتم (p(p)=log(Ns(p) وقيمة اللوغاريتم (p(p)

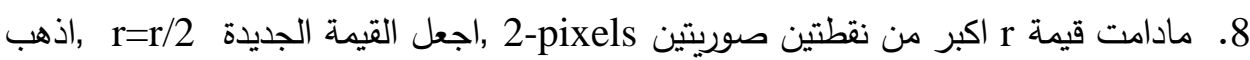

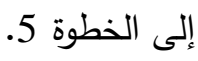

9. احسب ميل الانحدار الخطي لقيم المتجهين log(Ns) و (R)

$$
\text { المعادلتين : nen }
$$

$\mathrm{C}=$ Covariance $(\mathrm{A}, \mathrm{B})$

regslp $=\mathrm{C}(1,2) / \mathrm{C}(1,1)$
(9)

(10)

10. البعد ألكسوري يساوي المقدار (10)

يمثل الثكل(2) التكرارات الثلاثة الأخيرة لحساب البعد ألكسوري لساحل جزيرة بوبيان باستخدام

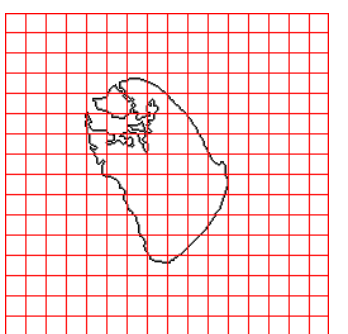

طريقة عد الصناديق

$\mathrm{N}=35$

$\mathrm{r}=21.5 \mathrm{~km}$

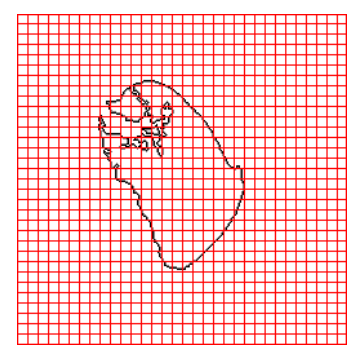

$\mathrm{N}=79$

$\mathrm{r}=10.25 \mathrm{~km}$

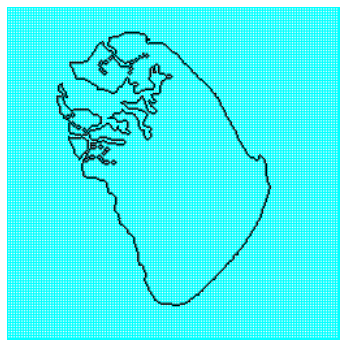

$\mathrm{N}=153$

$\mathrm{r}=5.125 \mathrm{~km}$

الثكل (2 )التكرارات الثلاثة الأخيرة لحساب البعد ألكسوري لساحل جزيرة بوبيان

باستخدام طريقة عد الصناديق,مقياس الرسم 1ملم =5.25 كم 


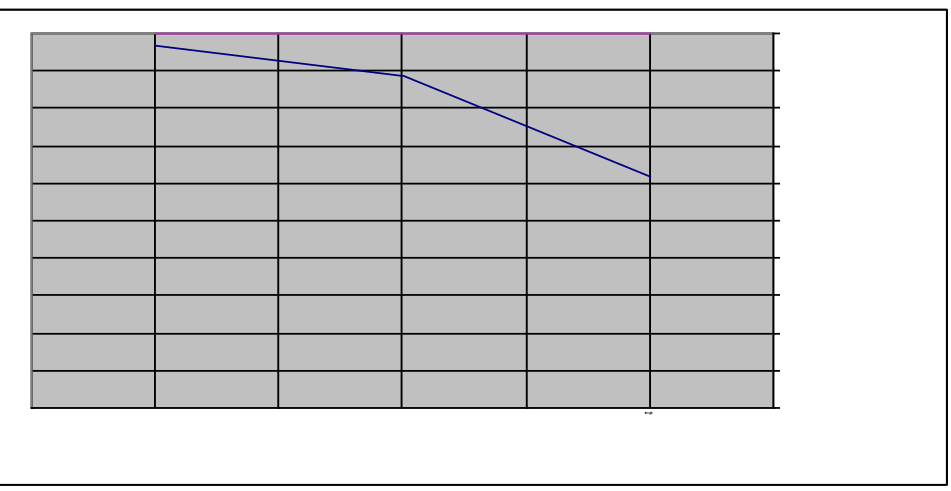

الثكل (3) يمثل الثكل الرسم البياني بين r و و

والخط في الثكل( 3 ) يناظر العلاقة في المعادلة (8), إذ إن البعد الكسوري لكنحني الشريط الساحلي هو" 18028" ويمكن حساب طول الشريط الساحلي من العلاقة:

$\mathrm{N}(\mathrm{r})=$ const. $\mathrm{r}^{1-\mathrm{DB}}$

$\mathrm{L}(\mathrm{r})=\mathrm{N}(\mathrm{r}) \cdot \mathrm{r}$

$=712 \cdot \mathrm{r}^{1.17028} \cdot \mathrm{r}$

$=712 . r^{1}{ }^{1.17028}$

$=$ const. $\mathrm{r}^{1-\mathrm{DB}}$

وبهذا يكون طول الثريط الساحلي تقريبا (562.807) كم رو ان 712تمثل عدد الصناديق

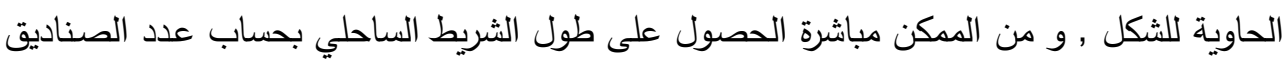

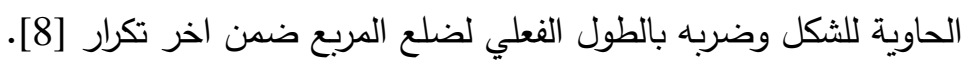

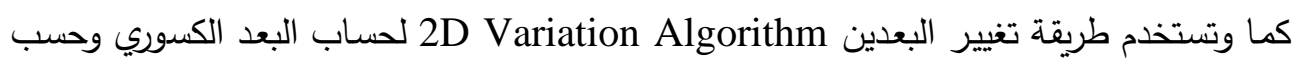

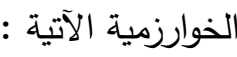

4. الخوارزمية (2) خوارزمية حساب البعد ألكسوري - طريقة تغيير البعدين:

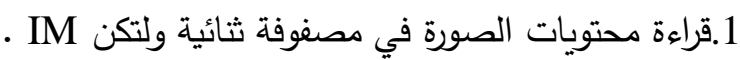

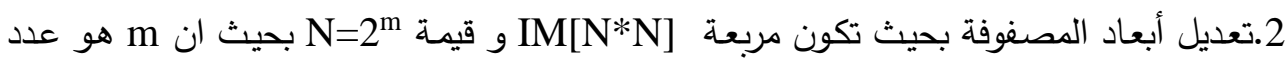

3. حساب نقطة مركز الصورة.

4.افتراض قسم المركز للصورة بمربع أبعاده 2x2 نقطة الصورة.

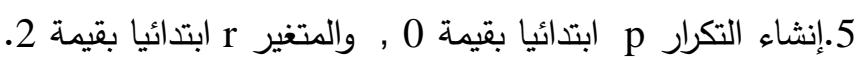

106 
6. افتراض مربع SQ طول ضلعه r من الأقسام المربعة طول ضلع كل مربع نقطتان يكون للمركز مربعً طول ضلعه نقطتان وزاويته السفلى اليمنى هي مركز الصورة.

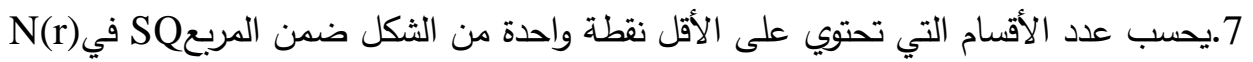
8. حساب (1/r) و N(r) للتكرار الحالي في المتجهين A و B م على التوالي.

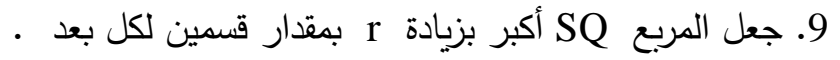

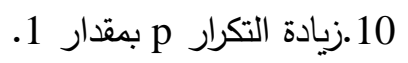

11. تكرار الخطوات من 3 إلى 10 حتى يغطي المربع SQ الصورة كاملة في اطار المصفوفة . $\mathrm{IM}$

12. حساب S ميل الخط المستقيم لقيم لوغاريتم الوسط الحسابي لA مقسوما على لوغاريتم الوسط

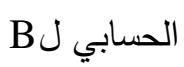
وحساب" D "الذي يمثل البعد ألكسوري و حسب المعادلتين:

$\mathrm{S}=\log (\mathrm{A}(\mathrm{p}) / \mathrm{p}) / \log (\mathrm{B}(\mathrm{p}) / \mathrm{p})$

$\mathrm{D}=2-\mathrm{S}$

تصلح هذه الخوارزمية لحساب البعد الكسوري لبيئة النقاط الصورية [8] رلمختلف القياسات (Scales)

$$
\text { : r=5,r=7and r=9 ) حيث (4) }
$$

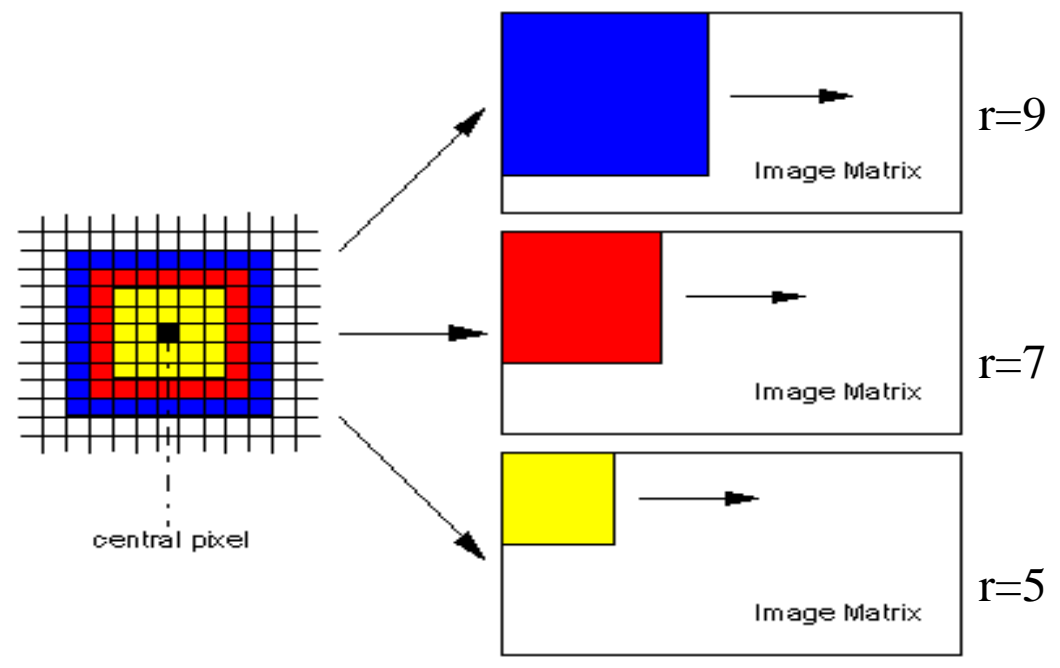

الشكل (4)تثثيل خوارزمية تغيير البعدين

ثم حساب"S" ميل المستقيم لقيم(Log(r) Log N(r) ثم استخراج البعد الكسوري من المعادلة : 
$\mathrm{D}=2-\mathrm{S}$

فضلاً عن حساب البعد ألكسوري لصور الخرائط والمنحنيات , يوفر برنامج البعد الكسوري

إمكانية حساب طول الحدود (Borders length) للخرائط والمنحنيات كل حسب مواصفاته , بحيث يضاف إلى الخرائط مقياس الرسم , ويكفي المنحنيات ان تمثيلها الصوري هو من دون تكبير , هذان التهات

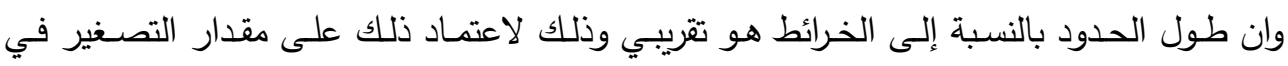

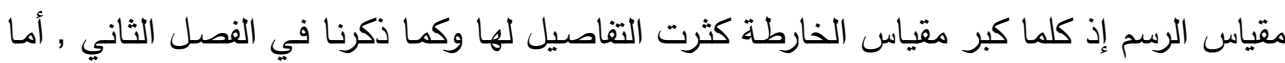

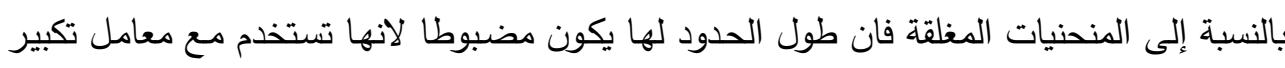
وليس معامل التصغير كما هو الحال لمقياس الرسم للخرائط الجغرافية و أدناه الخوارزمية : 5. الخوارزمية (3) خوارزمية حساب طول الحدود للخرائط والمنحنيات المغلقة من صورها الرقمية:

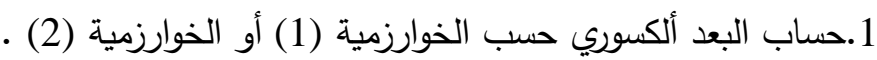
2.ضمن أخر تكرار في الحساب لقيم N(r) , ضع قيمr و N(r) النهائية في نظر الاهتمام . 3. احسب طول الحدود من المعادلة :

Length $=(\mathrm{N}(\mathrm{r}) \cdot \mathrm{r}) /(38)$

إن 38 هو عدد ثابت يمثل عدد نقاط خط مستقيم طوله سنتيمتر واحد. 4.ذا كان الاختيار لخارطة فان الناتج لطول الحدود النهائي يكون حسب المعادلة: MapBorderLength=Length* $1 /$ Scale

بحيث أن Scale هو مقياس الرسم لما يمثل من كيلومترات لكل سنتيمتر · 5.إذا كان الاختيار لحساب طول منحني مغلق فان طول حدوده هو : CurveBorderLength=Length*EnlargeScale بحيث أن EnlargeScale هو معامل التكبير للمنحني • ملاحظة: في 4 الناتج يكون مقاسا بالكيلومتر , وفي 5 الناتج يكون مقاسا بالسنتيمتر ·

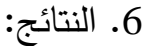
المثال (1): حسـاب البعد ألكسـوري لهيئـة حدود خارطـة العراق بمقيـاس رسم 1سم =200 كم للصورة داخل الثكل (5) بطريقة عد الصناديق المعدلة :

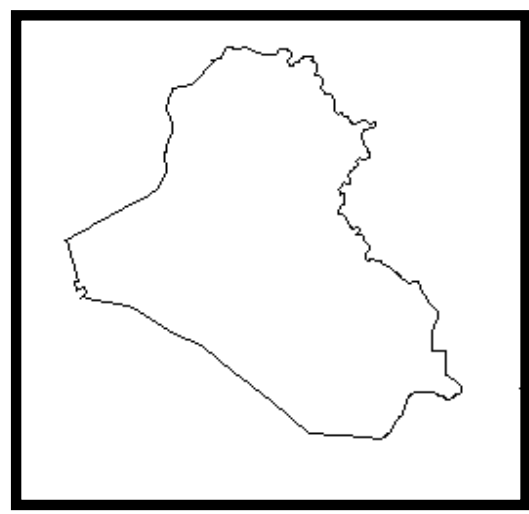

الثكل (5) صورة هيئة حدود خارطة العراق بمقياس رسم 1سم 
الجدول (1) تفاصيل التقطيع وحساب البعد ألكسوري للصورة المبينة في الثكل (5)

\begin{tabular}{|c|c|c|c|c|c|c|c|}
\hline \multirow[b]{2}{*}{$\log (N(r))$} & \multirow[b]{2}{*}{$\log (1 / r)$} & \multirow{2}{*}{ 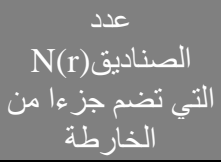 } & \multicolumn{3}{|c|}{ طول الضلع r } & \multirow[b]{2}{*}{ التصغير } & \multirow[b]{2}{*}{ التكرار } \\
\hline & & & بالفقاط & بالنسبة إلى 2m & الطول الابتدائي & & \\
\hline 0 & -7.205909 & 1 & 256 & $2^{0}$ & 1 & 0 & 1 \\
\hline 1.386294 & -6.512761 & 4 & 128 & $2^{-1}$ & 0.5 & -1 & 2 \\
\hline 2.484907 & -5.819614 & 12 & 64 & $2^{-2}$ & 0.25 & -2 & 3 \\
\hline 3.218876 & -5.126467 & 25 & 32 & $2^{-3}$ & 0.125 & -3 & 4 \\
\hline 4.025352 & -4.433320 & 56 & 16 & $2^{-4}$ & 0.0625 & -4 & 5 \\
\hline 4.727388 & -3.740173 & 113 & 8 & $2^{-5}$ & 0.03125 & -5 & 6 \\
\hline 5.446737 & -3.047026 & 232 & 4 & $2^{-6}$ & 0.015625 & -6 & 7 \\
\hline 6.037871 & -2.353878 & 418 & 2 & $2^{-7}$ & 0.0078125 & -7 & 8 \\
\hline
\end{tabular}

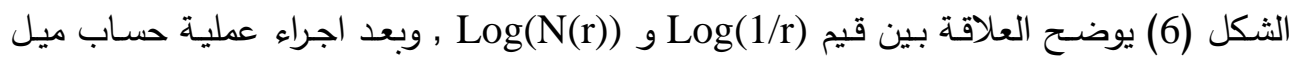

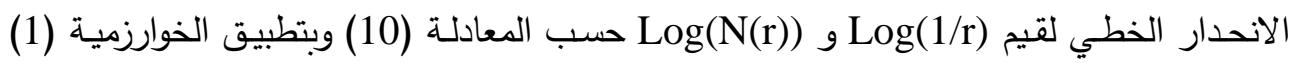
ينتج البعد ألكسوري الذي يساوي118428, التيتطبيق الخوارزمية(3) تم ايجاد الطول الممهد(قليل

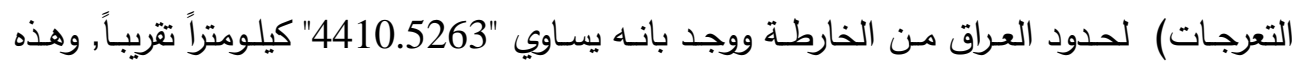
النتيجة مقاربة لطول حدود جمهورية العراق المعروفة لاى الجغرافيين والتي تساوي "4443 " كيلومتراً • أما إذا اعتبرنا ان الخارطة هي منحني مغلق وطبقنا الخوارزمية (3) فنجد أن طول المنحني يساوي "22.526" سنتيمتر وهو ما يمثل طول المنحني المغلق ضمن المقياس المستخدم لتمثيل

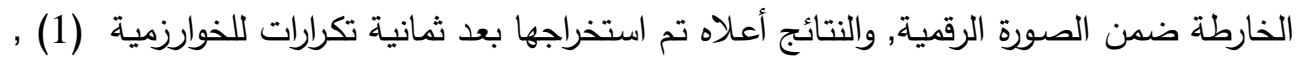

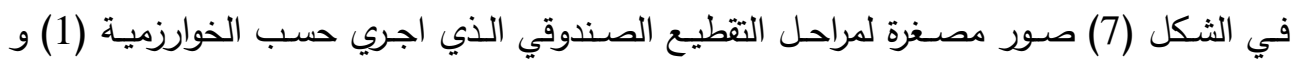

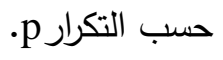



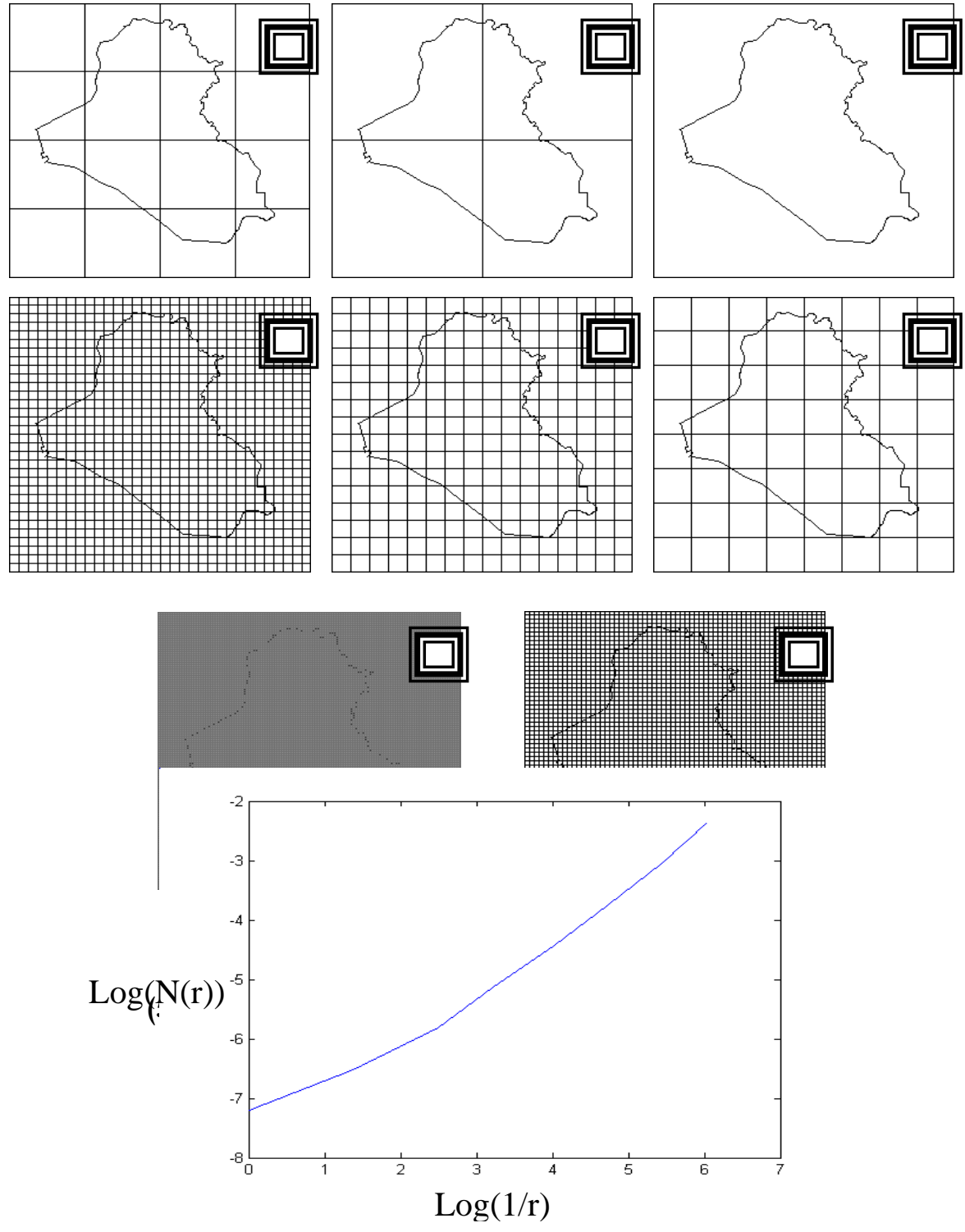

الثكل (6) العلاقة بين Log(1/r) و Log(N(r) Lلمثال (1)

وقد كانت الصور المستخدمة في هذا الثثال صورة رقمية من صيغة BMP التي تم تعديل أبعادها إلى 256x256 نقطة .لتتلاءم مع حسابات الخوارزمية . 
وبعد تطبيق الخوارزميـة (2) ينتج البعد ألكسوري الذي يسـاوي "1.25952 " وبتطبيق

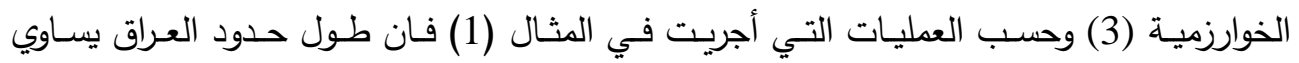

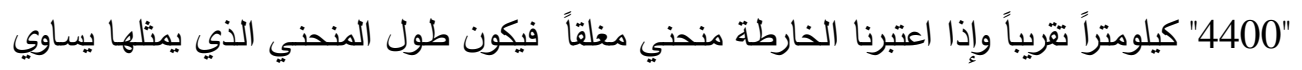
"22" سنتيمتراً, ويمكن الملاحظة أن هذه النتائج تكون في توافق مقبول مع النتائج التي تم الحصول

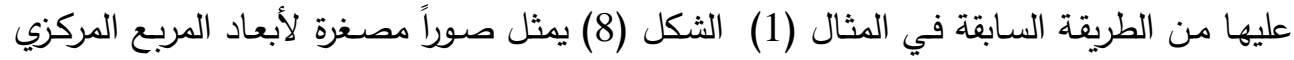

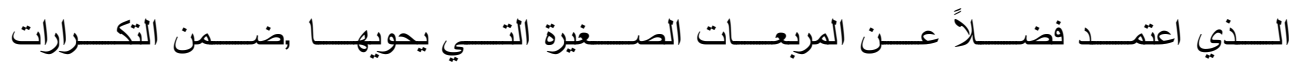
64,56,48,40,32,24,16,8,1

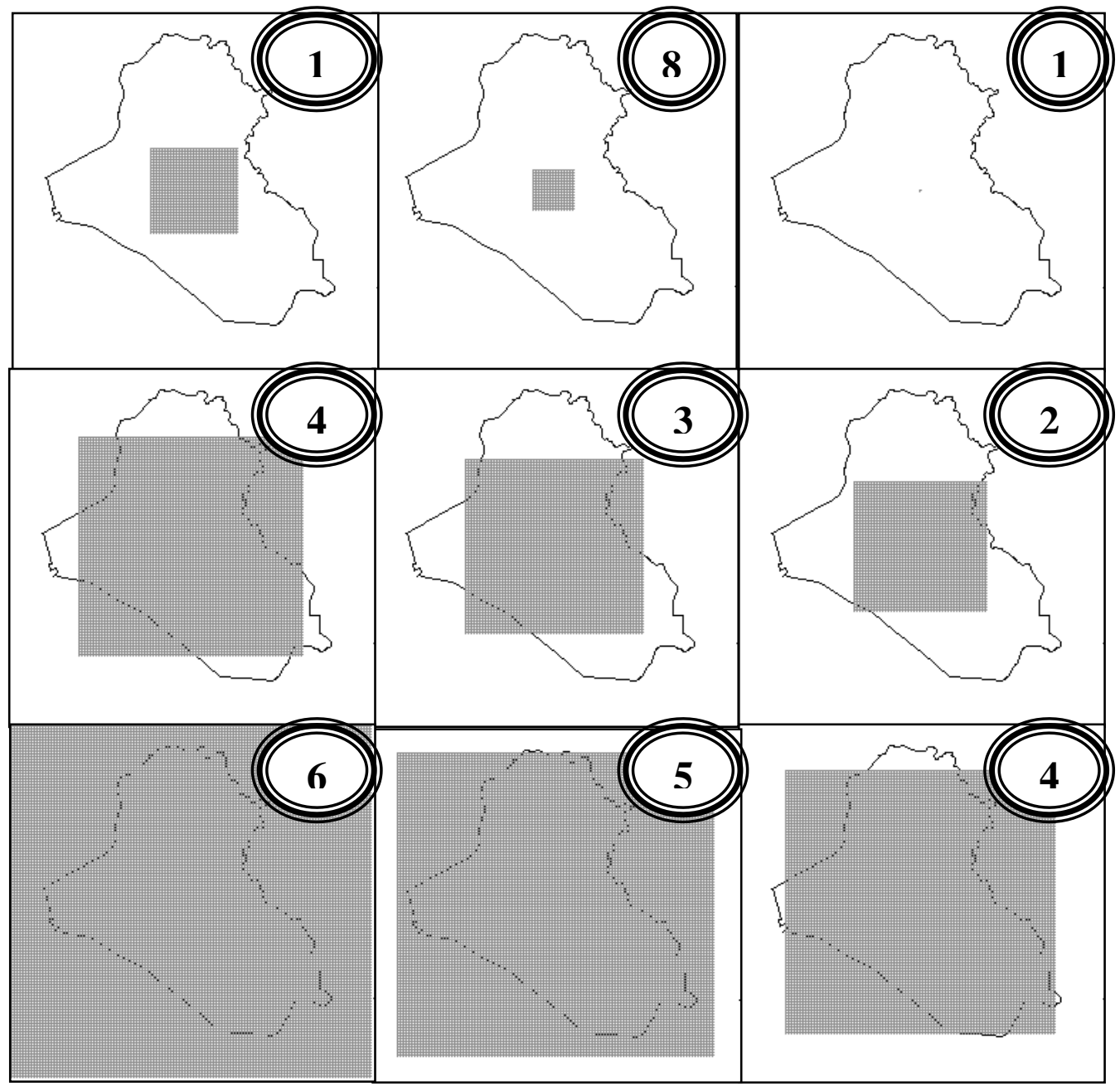

الشكل (8) صور مصغرة توضح أبعاد المربع المركزي الذي اعتمد فضلاً عن المربعات

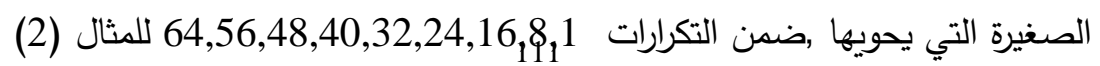


7. الاستنتاجات والتوصيات:

1.ان طريقة عد الصناديق أعطت نتائج أدق في الحساب وسرعة أعلى في التتفيذ مقارنةً بطريقة تغيير البعدين •

2.تمت ملاحظة فرق في البعد ألكسوري بين الطريقة الأولى والطريقة الثانية بواقع 6\% , وهو فرق مقبول من الناحية العملية.

3.في حالة مضاعفة مقياس الرسم للخرائط تتولد لنا زيادة في البعد الكسوري بمقدار 3\% م. 3.

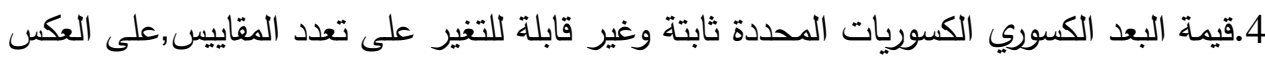
فان الكسوريات غير المحددة لا يكون البعد الكسوري لها ثابتاً. 5.تمكين البرنامج من معالجة مشكلة مقياس الرسم. 6.استخدام البرنامج لحساب مساحات الدول. 7.رفع إمكانية البرنامج للتعامل مع الأشكال ثلاثية الأبعاد بأخذ خرائط الدول تبعا للسطح "Surface" " وحساب البعد الكسوري لهيئة معالم وحدود سطح الدولة.

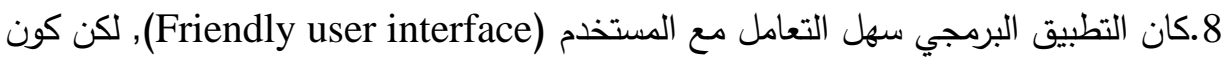

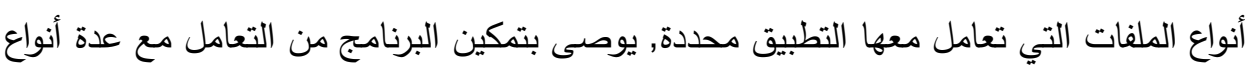

$$
\text { من الملفات الصورية. }
$$

9.استخدام البعد الكسوري عنصر تمييز لمدى انتشار شكل أو هيئة في بيئة ما.

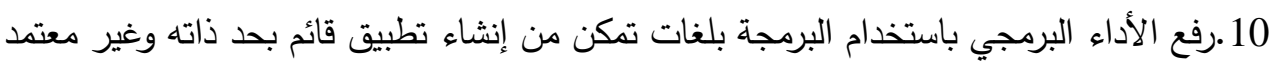
على تحميل مترجم اللغة البرمجية في الحاسبة. 


$$
\begin{aligned}
& \text { |لمصادر } \\
& \text { العبيدي ,انعام هادي عبد "دراسة عن الكسوريات وتطبيق هينون مع حالة دراسية في } \\
& \text { علم البيئة ",كلية علوم الحاسبات والرياضيات , جامعة الدوصل } 2002 \text { ـ }
\end{aligned}
$$

[2] Alligood,K.T,Sauer,T.D and Yourk,J.A."Chaos : An Introduction to Dynamical Systems ",Verlag New York Inc 1997.

[3] B.B. Mandelbrote "The Fractal Geometry of Nature " W.H FREEMAN Co. ,New York 1982 .

[4] K.Falconer "Fractal Geometry :Mathmatical Foundation and application, " John Wisly \& Sons ltd ,1990.

[5] M.F.Barnesly ,B.B.Mandelbrot , R.L.Devaney , H.Peitgen , D.Saup , R.F. Voss , Y.Fisher and M.M Mecuire " The Science of Fractal Images", Spriger Verlag ,New York, 1988.

[6] S.Herrington "Computer Graphics : A Programming Approach" $2^{\text {nd }}$ ed. 1987

[7] K.C. Clarke "Computation of topographic surfaces using the triangular prism surface area method ", Computers and Geosciences ,Vol 2 ,No 5 ,1986,P713-722.

[8] Roland Kraft, Josef Kauer "Estimating the fractal dimension from digitized images" Munch university of Technology-Weihenstephan Department of Agricultural and Horticultural Sciences Mathematics , Statistics and data Processing institute D-85350 Freising / Germany 1995. 